\title{
Implications of the Contact Radius to Line Step (CRLS) Ratio in AFM for Nanotribology Measurements
}

\author{
James M. Helt ${ }^{1 \dagger}$ and James D. Batteas ${ }^{2} *$ \\ ${ }^{1}$ Department of Chemistry, College of Staten Island and the Graduate Center of the City \\ University of New York, 2800 Victory Boulevard, Staten Island, NY 10314
}

and

${ }^{2}$ Department of Chemistry, Texas A\&M University, PO Box 30012, College Station, TX 77842

*Corresponding author: batteas@mail.chem.tamu.edu

${ }^{\dagger}$ Present address: The Aerospace Corporation, Micro/Nanotechnology Department, Space

Materials Laboratory, PO Box 92957, Mail Stop M2-271, Los Angeles, CA 90009-2957.

Phone: 310-336-0419

\section{Supporting Information}

Description of the model and derivation of the expressions used in the scan correction $\left(\operatorname{scan}_{c o r}\right)$ calculations.

The initial functional form defining the regions of overlapping Hertz contacts of radius $a$ is:

$$
\left(\frac{1.5 F_{z}}{\pi a^{2}}\right)\left[\int_{0}^{a \sin (\theta)} \int_{0}^{\sqrt{a^{2}-x^{2}}} p(x, y) d y d x-\int_{\alpha}^{a \sin (\theta)} \int_{0}^{\beta} p(x, y) d y d x+\int_{0}^{\chi} \int_{0}^{-\beta} p(x, y) d y d x\right] .
$$


As previously defined in the text and in Figure 3, the limits of integration $\beta$ and $\chi$ are:

$$
\beta=-\sqrt{a^{2}-x^{2}}+2 a \cos (\theta)
$$

and

$$
\chi=\sqrt{a^{2}-(2 a \cos (\theta))^{2}} .
$$

The three separate double integrals were evaluated independently as follows.

I. Integration of the first function in eq. (A1) -

$$
2\left(\frac{3 F_{z}}{2 \pi a^{2}}\right)^{\sqrt{a^{2}-x^{2}}} \int_{0}^{\frac{a^{2}-x^{2}}{a^{2}}-\frac{y^{2}}{a^{2}}} d y=2\left(\frac{3 F_{z}}{2 \pi a^{2}}\right)\left(\frac{\pi\left(a^{2}-x^{2}\right)}{4 a}\right)=\left(\frac{3 F_{z}}{4 a^{3}}\right)\left(a^{2}-x^{2}\right),
$$

which is then integrated with respect to $x$ to yield the analytic solution:

$$
\left(\frac{3 F_{z}}{4 a^{3}}\right)^{a} \int_{0}^{\sin (\theta)}\left(a^{2}-x^{2}\right) d x=\left(\frac{3 F_{z}}{4}\right)\left(\sin (\theta)-\frac{1}{3} \sin (\theta)^{3}\right) .
$$

II. Integration of the second function in eq. (A1) - 


$$
\begin{gathered}
2\left(\frac{3 F_{z}}{2 \pi a^{2}}\right)^{-\sqrt{a^{2}-x^{2}}+2 a \cdot \cos (\theta)} \sqrt{\frac{a^{2}-x^{2}}{a^{2}}-\frac{y^{2}}{a^{2}}} d y= \\
=\left(\frac{3 F_{z}}{2 \pi a^{3}}\right)\left[\sqrt{4 a \cos (\theta) \sqrt{a^{2}-x^{2}}-4 a^{2} \cos (\theta)^{2}} \times\left(-\sqrt{a^{2}-x^{2}}+2 a \cos (\theta)\right)+\left(a^{2}-x^{2}\right) \arcsin \left(\frac{2 a \cos (\theta)}{\sqrt{a^{2}-x^{2}}}-1\right)\right]
\end{gathered}
$$

Expression (A7) is then numerically evaluated with respect to $x$ :

$$
\int_{\alpha}^{a \sin (\theta)}(\text { eq. A7) } d x,
$$

where the integration limit $\alpha$ is dependent on the magnitude of angle $\theta$. For $\theta$ greater than $\pi / 3, \alpha$ is equal to $\chi$ as previously defined.

$$
\alpha=\begin{aligned}
& 0 \text { for }\left(\theta \leq \frac{\pi}{3}\right) \\
& \chi \text { for }\left(\theta>\frac{\pi}{3}\right)
\end{aligned}
$$

III. Integration of the third function in eq. (A1) -

$$
\left(\frac{3 F_{z}}{2 \pi a^{3}}\right)^{\sqrt{a^{2}-x^{2}}-2 a \cdot \cos (\theta)} \int_{0}^{\frac{a^{2}-x^{2}}{a^{2}}-\frac{y^{2}}{a^{2}}} d y=
$$




$$
=\left(\frac{3 F_{z}}{2 \pi a^{3}}\right)\left[\sqrt{4 a \cos (\theta) \sqrt{a^{2}-x^{2}}+4 a^{2} \cos (\theta)^{2}} \times\left(\sqrt{a^{2}-x^{2}}-2 a \cos (\theta)\right)+\left(a^{2}-x^{2}\right) \arcsin \left(1-\frac{2 a \cos (\theta)}{\sqrt{a^{2}-x^{2}}}\right)\right]
$$

Expression (A11) is then numerically evaluated with respect to $x$ :

$$
\int_{0}^{\sqrt{a^{2}-(2 a \cdot \cos (\theta))^{2}}}(\text { eq. A11) } d x
$$

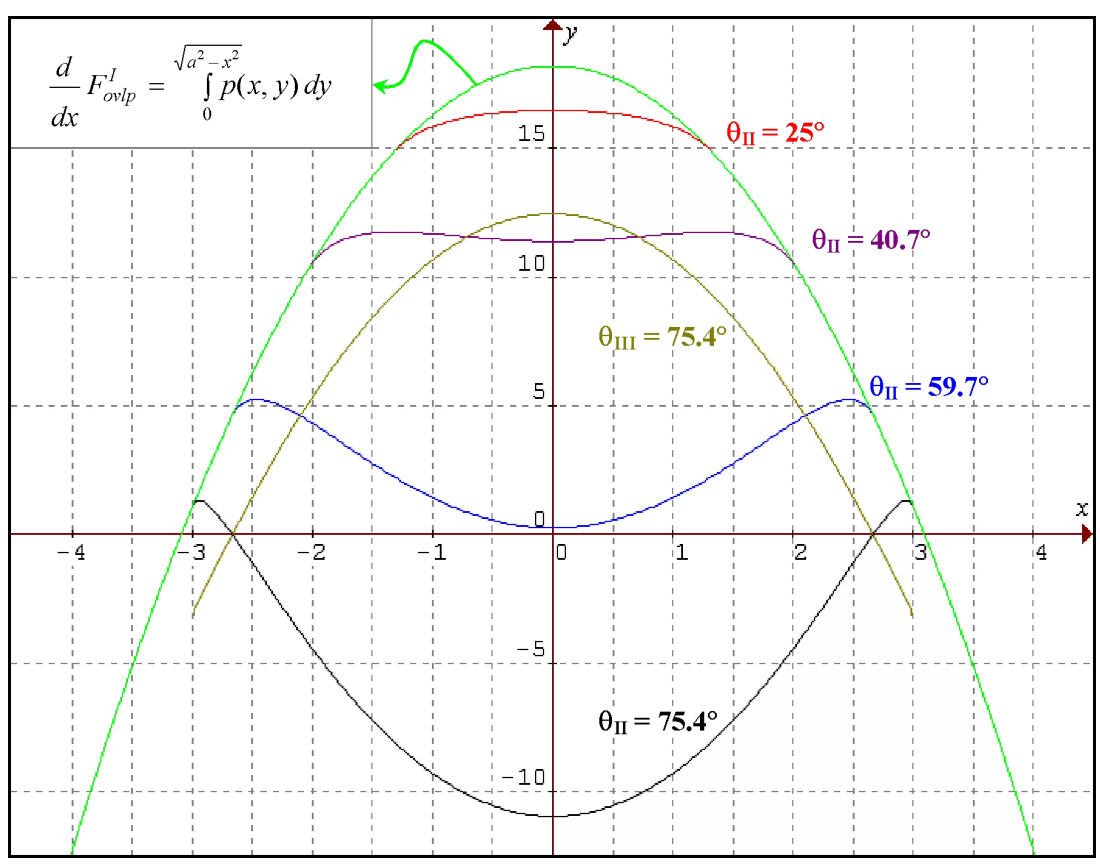

Figure S1. Graphical forms of eqs. (A4, A7, A11) after first integration for mica-silicon material pair. Here the load was $75 \mathrm{nN}$ corresponding to a Hertz mean pressure $\left(p_{m}\right)$ of $2.5 \mathrm{GPa}$. Experimental parameters were: $R=30 \mathrm{~nm}$, res $=256, L_{\text {sscan }}=400 \mathrm{~nm}$ and $L S=1.5625 \mathrm{~nm}$. The first term, $F_{\text {ovl }}^{I}$ given in eq. (A4), after first integration is independent of $\theta$ and is marked with the arrow. All functions defined with $\theta_{\text {II }}$ are forms of the second term ( $F_{\text {ovlp }}^{I I}$, eq. A7) after the first integration for the given angle. $\theta_{\mathrm{III}}$ is representative of the third term ( $F_{\text {ovlp }}^{I I I}$, eq. A11), which is only evaluated for $\theta>60^{\circ}$. 


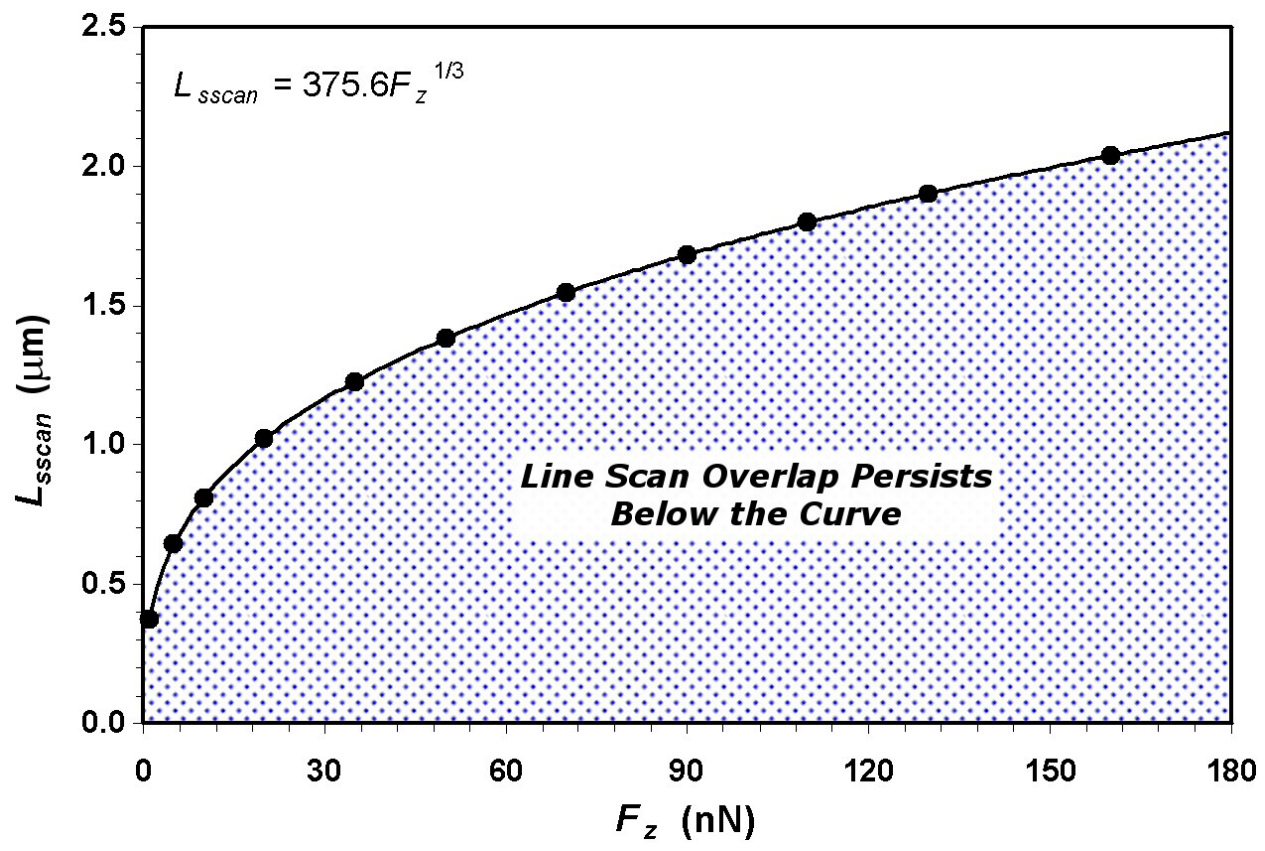

Figure S2. Projected slow scan length, $L_{\text {sscan }}^{C R L S}$ required to avoid line scan overlap for a silicon tip $(R=30 \mathrm{~nm})$ and a mica substrate.

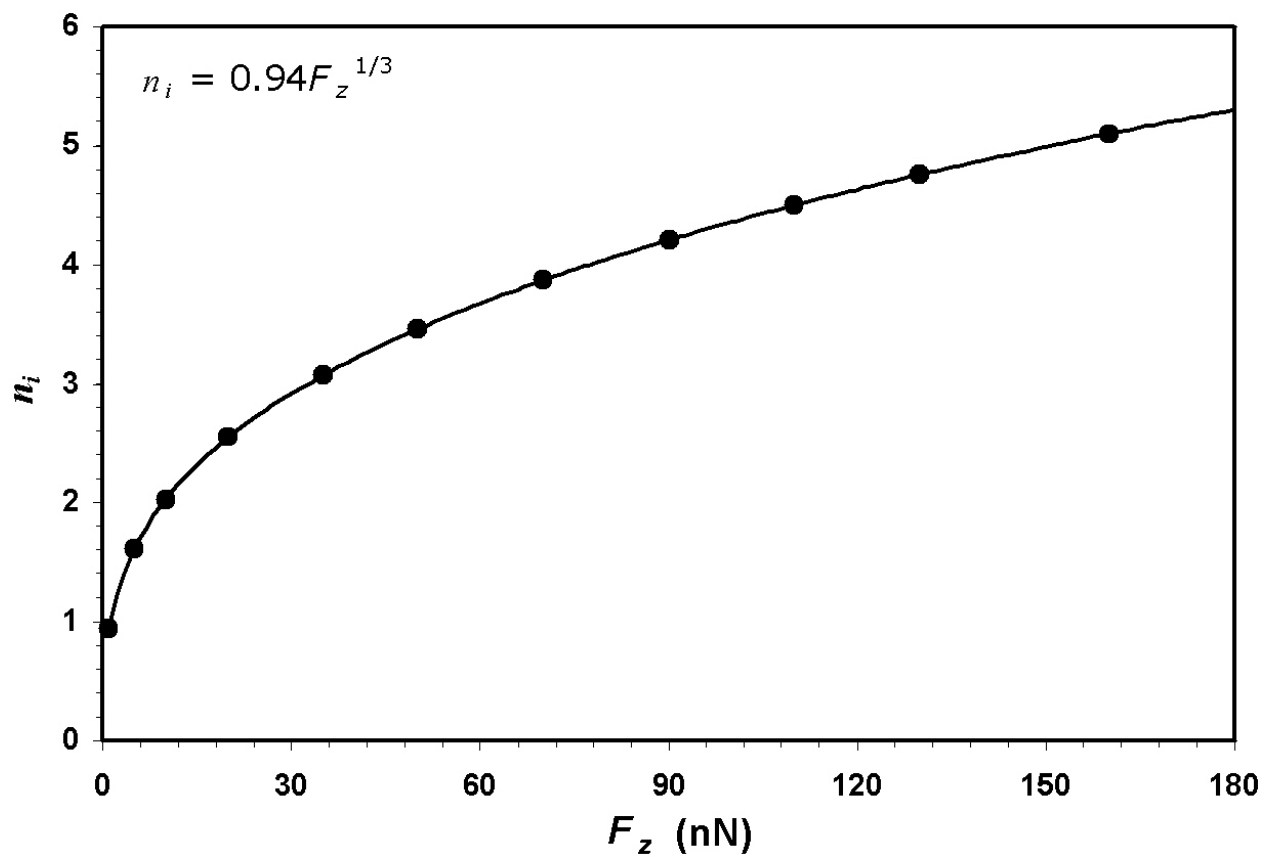

Figure S3. Projected truncating parameter $n_{i}$, as defined by eq. 12, in the text for the siliconmica material pair. $n_{i}$ indicates the number of successive line scans with scan overlap and will therefore contribute to the unaccounted contact history that is estimated by $\operatorname{scan}_{\text {cor }}$. 


\section{Modeling Scan Overlap Data}

For the mica-Si material pair, the $L_{s s c a n}^{C R L S}$ vs. $F_{z}$ plot given in Figure S2, indicates that ideal, non-overlapping AFM wear trials over a $(400 \times 400) \mathrm{nm}^{2}$ scan area can only be achieved under very low applied loads. Similarly, Figure S3 illustrates the expected truncating parameters $\left(n_{i}\right)$ proportionality to load $\left(n_{i} \propto F_{z}^{1 / 3}\right)$, indicating that the number of successive line scanscontributing unaccounted scan history to the surface escalates non-linearly with load. Figures S2 and S3 are instructive and clearly demonstrate that the systematic error associated with this geometric-scanning effect can pervade AFM nanotribology data sets. As outlined by the CRLS analysis, this systematic error is addressable and can be quantified in terms of a scan cor. A analytical solution of eq. 8(b) in the text over all $\theta$ is not available and therefore $\operatorname{scan}_{\text {cor }}$ was evaluated numerically for a wide range of loads, including those summarized in Table S1. Figure S4 illustrates the expected shifted power law dependence $s c a n_{c o r}$ has on $F_{\mathrm{z}}$, eq. A13, for the mica-silicon material pair. The interpretation of $s c a n_{c o r}$ is straightforward. Referring to Table S1, under a load of $75 \mathrm{nN}$, scan cor $_{\text {was }}$ found to be 2.29, indicating that a single image scan imparts 2.29 scans worth of scan history to the surface. Calculations also show that even for a load of $5 \mathrm{nN}$, scan $c$ cor expressed a $25 \%$ departure from the experimental number of scans value

\begin{tabular}{|c|c|c|c|}
\hline $\boldsymbol{F}_{z}(\boldsymbol{n} N)$ & $a(\mathrm{~nm})$ & $n_{i}$ & $\operatorname{scan}_{\text {cor }}$ \\
\hline 1 & 0.73 & 1 & 1.00 \\
\hline 5 & 1.25 & 2 & 1.25 \\
\hline 10 & 1.58 & 3 & 1.41 \\
\hline 25 & 2.14 & 3 & 1.76 \\
\hline 75 & 3.09 & 4 & 2.29 \\
\hline 150 & 3.90 & 5 & 2.75 \\
\hline 400 & 5.41 & 7 & 3.62 \\
\hline
\end{tabular}

Table S1. Parameters and results from the line scan overlap calculations. 


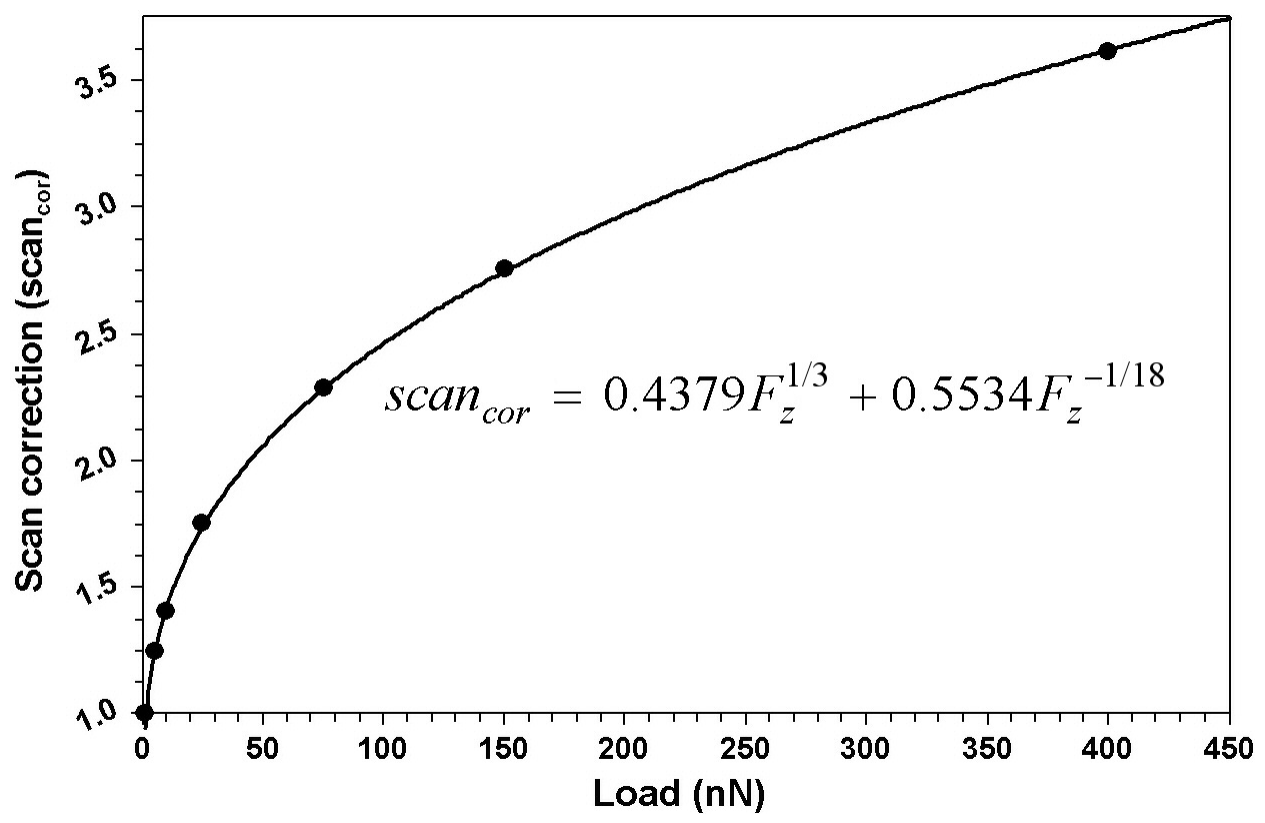

Figure S4. Numerical results of eq. $8 \mathrm{~b}$ for $\operatorname{scan}_{\text {cor }}$ (filled circles in plot). AFM image parameters were res of $256(L S=1.5625 \mathrm{~nm})$ and $L_{\text {sscan }}$ of $400 \mathrm{~nm}$ for a silicon AFM tip $(R=30 \mathrm{~nm})$ and a mica substrate. Linear regression analysis (solid line) finds that $\operatorname{scan}_{\text {cor }}$ follows a shifted power law dependence with $F_{z}$.

(Table S1). These results provide compelling insight into the actual history experienced by the surface and prompts new questions into how this additional scan history affects defect growth and interpretation of extracted kinetic parameters.

$$
\operatorname{scan}_{\text {cor }}=0.4379 \times F_{z}^{1 / 3}+0.5534 \times F_{z}^{-1 / 18}
$$

Although one can extrapolate the correction for a range of loads with this expression, it is not a universal solution since the variables, namely res, $L S$ and the material dependent $a$, are part of the fit. According to this Hertzian development, a percent force overlap vs. $\theta$ plot, Figure S5, is a universal overlap correction curve and is applicable to any tip-substrate material pair. This general overlap correction curve is realized by taking advantage of the normalized $\theta-a$ relationship derived from the Hertz contact geometry, given in the text by eq. (12), and by leaving the $m$ and $r e s$ variables for the final summation. Thus, the general $s_{c a n}$ cor form is given 


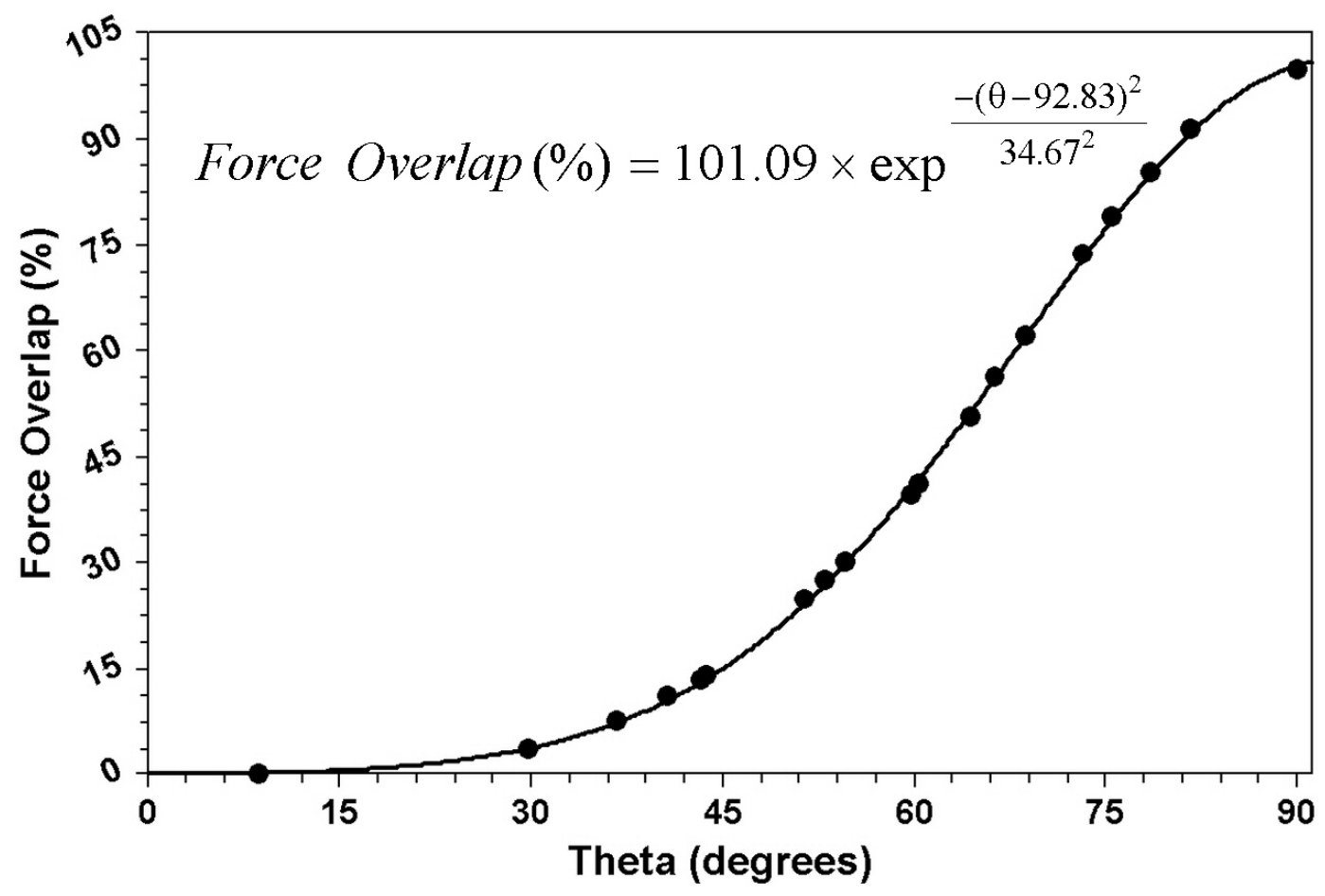

Figure S5. General form of the overlap correction curve for all tip-substrate material combinations for estimating $s c a n_{c o r}$. Linear regression analysis (solid line) finds that the percent force overlap grows exponentially with $\theta$.

as a function of $(\theta, m)$ where both parameters have pure geometric origins centered on $a$. One can then readily calculate $\theta(m)$ and simply sum over the range of angles to get $s c a n_{c o r}$ via eq. (A13). Non-linear regression analysis finds a standard error of 0.48 for the fit in Figure S5, which corresponds to a few percent error in the calculated $s_{c a n}$ cor. Thus, eq. (A14) is a reasonable approximation of eq. (8b) in the text and provides a straightforward expression to implement the CRLS methodology for all tip-substrate material pairs.

$$
\operatorname{scan}_{c o r}(\theta, m)=1+\sum_{\theta\left(m_{1}\right)}^{\theta\left(m_{i}\right)}\left[\frac{r e s-m}{r e s}\right] \times\left(1.0109 \times \exp ^{\frac{-(\theta-92.83)^{2}}{34.67^{2}}}\right)
$$

